\title{
Los estudios de la India en Latinoamérica. Desarrollo, integración y perspectivas futuras
}

\author{
Gabriel Martino ${ }^{1}$ \\ Universidad de Buenos Aires; Conicet (Argentina) \\ Julio César Ossa² \\ Universidad de San Buenaventura (Colombia)
}

Recibido: febrero 15 de 2015 - Revisado: marzo 25 de 2015 - Aceptado: abril 20 de 2016

Referencia formato APA: Martino, G., \& Ossa, J. C. (2016). Los estudios de la India en Latinoamérica. Desarrollo, integración y perspectivas futuras Rev. Guillermo de Ockham, 14(1), 17-23. doi: http://dx.doi.org/10.21500/22563202.2404

\section{Resumen}

La publicación en América Latina de un número monográfico sobre estudios de la India es un fenómeno académico con una significación sociológica y política muy interesante. Es evidencia del encuentro entre dos regiones periféricas respecto de los centros científicos y académicos mainstream más prestigiosos de Europa y Norteamérica. ${ }^{3}$ Es cierto que si bien tal encuentro procura la producción y promoción propia (y colaborativa) de conocimiento, no carece de signos de dependencia académica respecto de los mencionados centros. Un relevamiento de la bibliografía utilizada por los especialistas que participan de nuestro número puede brindarnos una noción del grado de tal dependencia. No obstante, este encuentro también es un claro índice de la voluntad y de la capacidad de nuestras regiones para el establecimiento de vínculos académicos bilaterales.

Palabras clave: Estudios de la India, universidad, filosofía, Latinoamérica

\section{India Studies in Latin America. Development, integration, and future perspectives}

\section{Abstract}

The publication in Latin America of a monographic issue about India Studies is an academic phenomenon with a very interesting sociological and political significance. It is an evidence of the encounter of two peripheral regions regarding the most prestigious scientific and academic mainstream institutions of Europe and North America. It is true that even though this encounter aims at the production and promotion of knowledge it does not lack signs of academic dependence regarding the centers mentioned. A study of the bibliography used by the specialists who participate in our issue can provide a notion of the degree of this dependence. Nevertheless, this encounter is also a clear index of the will and the capacity of our regions to establish bilateral academic relations.

Keywords: India Studies, university, philosophy, Latin America

1. Licenciado y Doctor en Filosofía por la Universidad de Buenos Aires. Becario posdoctoral Conicet. Profesor a cargo de la materia Historia de la Filosofía Antigua, Facultad de Filosofía, Letras y Estudios Orientales de la Universidad del Salvador, y adscripto a la cátedra de Sánscrito de la Facultad de Filosofía y Letras, Universidad de Buenos Aires. Mail: gabriel.filosofia@hotmail.com

2. Profesor de la Facultad de Psicología, Universidad de San Buenaventura. E-mail: jcossa@usbcali.edu.co, juceossa@gmail.com

3. Para una discusión acerca de las nociones de periferalidad y de centralidad o mainstream en el Sistema Académico Mundial véase Beigel y Salatino (2015: 11-36). Cfr., asimismo, Beigel (2010, 2013). 


\section{Os estudos da India em Latinoamérica. Desenvolvimento, integração e perspectivas futuras}

\section{Resumo}

A Publicação em latino-americana de uma edição especial sobre Estudos da india é um fenômeno acadêmico com um significação sociológica e política muito interessante. É evidência do encontro entre duas regiôes periféricas com relação dos centros cientistas e académicos mainstream mais prestigiados de europeus e norte-américa. É certo que, enquanto tal encontro visa a produção e promoção própria (e colaborativo) de conhecimento não é sinai de dependência acadêmica sobre os mencionados centros. Uma pesquisa da literatura utilizada por especialistas que participam de nossos número podem dar-nos uma ideia da extensão de tal dependência. No entanto, esta reunião também é uma indicação clara da vontade e da capacidade de nossas regióes para o estabelecimento de laços académicos bilaterais.

Palavras-chave: Estudos da India, universidade, filosofia, Latinoamérica

\section{Introducción}

La profunda influencia que las agendas y las metodologías características de los circuitos académicos centrales ejercen sobre esta clase de trabajos, hacen que instancias como el presente número monográfico den lugar a nuevas maneras de encarar las investigaciones mediante la definición de objetivos, estándares, metodologías y representaciones definidas por las necesidades y las perspectivas propias de los centros involucrados en el diálogo. Cabe señalar, no obstante, que nuestra labor no es en absoluto pionera. Tal como la doctora Flora Botton afirmó en una de sus contribuciones en el último Congreso Internacional de Aladaa, "un congreso de estudios de Asia en América Latina es consecuencia del trabajo de pioneros como Fernando Tola”. ${ }^{4}$ La investigación en el campo de los estudios de la India, de hecho, se desarrolla en Latinoamérica hace ya unas cuantas décadas. Diferentes centros universitarios, instituciones, programas, encuentros académicos y publi- caciones científicas dan cuenta de este hecho. El Centro de Estudios de Asia y África (Colmex, México), ${ }^{5}$ el Centro de Estudios Orientales (PUCP, Perú), la Fundación Instituto de Estudios Budistas (FIEB, Argentina) ${ }^{6}$ y la Escuela de Estudios Orientales (USAL, Argentina), ${ }^{7}$ por mencionar unos pocos, son centros académicos de grado, posgrado e investigación que vienen desarrollando una intensa e ininterrumpida actividad en el área por más de cuatro o cinco décadas. ${ }^{8}$ Numerosas cátedras y grupos de trabajo en distintas universidades también acompañan este fenómeno ${ }^{9}$ y la Asociación Latinoamericana de Asia y África (Aladaa) continúa desde hace cuarenta años su tarea de nexo y de promoción del trabajo de los especialistas en el área radicados en la región. ${ }^{10}$ La labor académica de los últimos años, no obstante, y la integración y colaboración entre los investigadores no ha conocido con anterioridad un grado tan intenso. ${ }^{11}$ Huelga decir que de modo paralelo a la actividad académica en los últimos años también se ha generado una enorme oferta

4. Véase la semblanza a Fernando Tola incluida en el presente número. Véase, asimismo, Tripathi (2012), quien hace una reseña de los estudios sánscritos fuera de India en el período 1950-2010. De Latinoamérica el autor solo menciona a la Argentina y de este país, a los especialistas F. Tola y C. Dragonetti.

5. Además de su intensa labor formativa, el Colmex desarrolla una importante tarea editorial de publicación de libros relativos a los estudios de la India y publica desde 1966 su prestigiosa revista Estudios de Asia y África.

6. Además de sus cursos regulares y de su labor investigativa, el FIEB publicó junto con la Asociación Latinoamericana de Estudios Budistas (ALEB) la Revista de Estudios Budistas editada en México y Buenos Aires entre los ańos 1991 y 1998.

7. Gran parte de las investigaciones llevadas a cabo en la escuela fue publicada en la Revista Oriente - Occidente, que se editó entre 1980 y 1999 . A partir del año 2001 y hasta el 2009 se edita la revista electrónica Transoxiana, Revista de Estudios Orientales.

8. Para un análisis de las cátedras dedicadas al estudio de filosofía de la India en Argentina véase Martino (2015). No podemos dejar de mencionar asimismo, el Museo Nacional de Arte Oriental (Buenos Aires, Argentina) que hace cinco décadas lleva a cabo su objetivo de difusión del conocimiento de las culturas asiáticas con el deseo de fomentar la comprensión entre los pueblos del mundo a través de exposiciones de variada temática e índole.

9. Al final de nuestra editorial brindamos un listado de las unidades académicas dedicadas al Estudio de la India en América Latina. Tal listado no es exhaustivo y constituye una instancia preliminar de una base de datos en preparación. Si desea informarnos acerca de su cátedra, centro o institución por favor comuníquese por mail con Gabriel Martino a gabriel.filosofia@hotmail.com

10. Considérese, asimismo, que tras la realización de las primeras jornadas internacionales sobre India que tuvieron lugar en Buenos Aires el 4 y 5 de septiembre de 2015, distintos especialistas acordaron constituir la Asociación Latinoamericana de Estudios de la India.

11. Mencionemos, a modo de ejemplo, que en 2015 se realizaron por primera vez tanto las Primeras Jornadas Internacionales sobre India (Buenos Aires, 3 y 4 de septiembre) como las Primeras Jornadas Internacionales de Filosofía Oriental (São Paulo, FFLCH/USP 21 al 23 de septiembre) en las que la India tuvo un espacio preponderante. Ambos eventos académicos reflejan la intensidad con que estos estudios se realizan en la región y el hecho de que, contando con antecedentes exitosos de carácter nacional, se hayan logrado organizar jornadas con carácter internacional en ambos países. En Chile, asimismo, se organizó el XV Congreso Internacional Aladaa (11 al 14 de enero 2016) en el cual la India fue el eje de numerosos paneles. Este último evento, además de celebrar los 40 ańos de Aladaa, puso de manifiesto la pertinencia, el impacto y la identidad propia que los estudios de la India tienen en América Latina.

$18<$ Universidad de San Buenaventura, Cali - Colombia 
de actividades, talleres, eventos culturales y comerciales en los que la India y diversos aspectos de sus identidades son puestos de relieve. ${ }^{12}$ El presente número, pues, es el resultado del nivel creciente de interés general y de la actividad integrada de los investigadores en las ciencias sociales y humanas de Latinoamérica dedicados a los estudios de la India. ${ }^{13}$

Vale la pena poner de manifiesto, por otra parte, la relación entre el progresivo desarrollo de los estudios de la India en nuestra región y el fortalecimiento de los vínculos económicos, comerciales y políticos entre India y Latinoamérica. ${ }^{14}$ Es evidente, asimismo, el creciente interés de parte de India por América Latina y que nuestra región ha dejado de ser un "continente olvidado" para aquella. ${ }^{15}$ Estos fenómenos han comenzado a ser estudiados por los especialistas. La bibliografía, de hecho, nos brinda un interesante reflejo de esta incipiente situación. En 2009 Lederman (Chile), Olarreaga (Uruguay) y Perry (Colombia), han editado el primer libro en inglés que analiza pormenorizadamente y de modo empírico los detalles de las relaciones económicas entre Latinoamérica e India (y China) en el siglo veintiuno, brindando con ello un importante punto de partida para futuras contribuciones en este campo. ${ }^{16}$ En 2012 el Foreign Policy Research Centre de India en Nueva Delhi editó posiblemente el primer número monográfico colaborativo entre especialistas indios y latinoamericanos dedicado a los estudios de las relaciones políticas, financieras, económicas y académicas entre estas regiones. ${ }^{17}$ En el mismo año, Heine publica en Chile La nueva India, descrito por Rengaraj Viswanathan del Centre for International Governance Innovation (CIGI) como el primer libro latinoamericano en español acerca del surgimiento de India en el siglo veintiuno. ${ }^{18} \mathrm{El}$ volumen contiene miradas sobre temas microeconómicos, macroeconómicos y de management con una perspectiva histórica, cultural y política. Un año después, Shidore (India) publica para el Indian Council for World Affairs un volumen dedicado al análisis de las tendencias en los vínculos de India con Latinoamérica en materias de economía, política, defensa y cultura. Su libro está dirigido a los agentes políticos en India que buscan capturar los aspectos vitales de la relación, así como a los de Latinoamérica que desean familiarizarse con el socio asiático de rápido crecimiento. ${ }^{19}$

Así pues, los distintos estudios coinciden en que se produjo un importante vuelco en la interacción directa de los países de Latinoamérica y la India en la década de los noventa, en la cual la cooperación comenzó a activarse de la mano de algunas políticas favorables para el crecimiento del comercio y del flujo de inversiones entre ambas regiones. ${ }^{20}$ Sin duda, resulta atractivo y prometedor para la relación India-Latinoamérica el hecho de que no haya un vínculo colonial entre ellas ni una estructura jerárquica. ${ }^{21}$ Los especialistas subrayan, por el contrario, la semejanza en los problemas de desarrollo que ambas regiones enfrentan, a pesar de las diferencias en sus trasfondos culturales. ${ }^{22}$ No obstante, uno de los mayores desafíos aparte de la distancia posiblemente sea el desconocimiento mutuo. "Resulta difícil proponer ideas de negocios e inversiones si no se conoce acerca de las oportunidades disponibles o no se posee el entendimiento contextual para observar las oportunidades en el mercado", afirma Burges. ${ }^{23}$ Incluso, las percepciones comunes que un sector posee del otro están sesgadas y basadas mayormente en mitos que ambos heredaron de sus regentes coloniales europeos, afirma Singh. "La impresión más común acerca de América Latina, añade, era que se trata de una región de gauchos, mariachis y carnavales. America Latina, por otra parte, percibía la India como una tierra de hombres sagrados, encantadores de serpientes y

12. No podemos dejar de mencionar el ya tradicional programa radial Niketan, Hogar de la India en Argentina, sobre arte, música, danzas, espiritualidad, cultura y empresas de la India en Argentina con la conducción de Gustavo Canzobre. El programa se emite los días lunes de 17 a 18 hs. por FM RADIO CULTURA 97.9, Buenos Aires, o por internet en vivo por http://www.fmradiocultura.com.ar/

13. Mencionemos también la Revista Cultura Oriental editada desde 2014 por la Universidade Federal da Paraíba.

14. Para un análisis pormenorizado de los vínculos económicos, comerciales y políticos entre India y Latinoamérica véase Rubiolo y Baroni (2014).

15. Gaur y Gaur (2012, p. 3). Para un temprano análisis de los incipientes estudios latinoamericanos en India véase Narayanan (1983, pp. 179-184). Tharoor (2012, p. 69) afirma que "Una parte del mundo que está comenzando a atraer una atención creciente es Latinoamérica. Ha sido desde largo tiempo el "continente olvidado" para la India - una región con la cual India podría haber encontrado muchos puntos en común pero, al estar separados por la distancia, el lenguaje y la carencia de una historia común de interacción, no lo hizo. Sin embargo [...] Latinoamérica claramente es una parte del mundo que los actores políticos de la India no pueden permitirse descuidar." Hacia el final de su artículo (p. 74) el autor afirma que "La relación India-LAC podría ser el ejemplo más interesante de la transformación del concepto todavía poco desarrollado de cooperación Sur-Sur".

16. Lederman, Olarreaga, y Perry (2009). Considérense, asimismo, Perry (2006), y Olarreaga y otros (2010, pp. 446-473).

17. Gaur y Gaur (2012).

18. https://www.cigionline.org/articles/2012/12/la-nueva-india-new-india-book-jorge-heine consultado el 5 de marzo de 2016.

19. Shidore (2013, p. 6).

20. Heine (2012, p. 6). Considérese, asimismo, la comparación que Solís (2001: 891-900) realiza entre México y la India, indicando que en la década de los noventa en ambos países ocurre una liberalización de la economía que condujo, hacia fines de la década, a una derechización en el ámbito político. En un trabajo posterior (2008, pp. 487-493) examina algunos puntos de diferencia entre los dos países como su desarrollo económico sus posturas frente al desarme nuclear.

21. Mesquita-Moreira (2012, p. 12).

22. Gaur y Gaur (2012, p. 3). También Shidore (2013, p. 51).

23. Burges (2012, p. 20). 
mendigos." ${ }^{24}$ Así pues, la tarea de producción, promoción y difusión del conocimiento mutuo, desligado hasta cierto punto de intereses foráneos a este vínculo resulta de vital importancia y ha comenzado a ser explorada. Considérese, además de las mencionadas publicaciones, la realización del primer India-LAC Investment Conclave en 2014 en Nueva Delhi, India, bajo el lema "Crezcamos juntos" que expresa el nuevo paradigma dentro del cual las relaciones India-LAC buscan establecerse. Pero tal cometido no debe llevarse a cabo solo en el ámbito de la política, la economía y las finanzas sino, también, en el terreno de la sociedad, la cultura y la historia en cuanto factores determinantes en las áreas primero mencionadas. Sin embargo, tal como afirman Burges y Singh, ambos aspectos de la relación no han entrado suficientemente en diálogo todavía. La búsqueda de gestiones políticas y financieras exitosas en las relaciones indolatinas no parece poder desestimar el conocimiento por parte de cada sector de los condicionantes sociohistóricos y culturales que operan en el sector socio. Vale la pena subrayar, de todos modos, que el establecimiento de este diálogo no implica colocar las investigaciones en ciencias sociales y humanidades dedicadas a los estudios de la India en el marco exclusivo de agendas financieras.

Consideramos que la situación que describimos es un indicador relevante de que a pesar de la gran dependencia académica que los estudios de la India en América Latina presentan respecto de las investigaciones producidas en centros europeos y norteamericanos, las investigaciones llevadas a cabo en nuestra región también pueden estar motorizadas por fenómenos locales y de interacción bilateral. Pensamos que esta coyuntura puede ser sumamente favorable para el desarrollo de perspectivas indolatinas. Ella podría propiciar, ciertamente, el desenvolvimiento de nuevas miradas y construcciones acerca de la India y de Latinoamérica que entren en diálogo no solo con las representaciones que cada región ofrece de sí misma, sino también con las representaciones que de ellas brindan los centros financieros y académicos norteamericanos y europeos. Sin embargo, la cooperación indolatina en materia académica y, sobre todo, en las áreas de ciencias sociales y humanas es una oportunidad que ambos agentes no se han dedicado a explorar y desarrollar todavía más que tímidamente. En un estudio en el que analiza la relación entre las universidades de India y Brasil y brinda propuestas para la implementación de cooperación académica entre ambos sectores, Leite afirma que tal trabajo cooperativo se encuentra en una etapa muy incipiente y que debe continuar en los próximos años de modo que ambas regiones puedan experimentar un crecimiento en conjunto. ${ }^{25}$

Creemos que un interesante índice de la apertura de ambas regiones al conocimiento mutuo y al intercambio es el nuevo proyecto del cineasta Pablo César. El director argentino ha comenzado a filmar en 2016 su nueva película coproducida con la India Pensando en él, en la que aborda la relación de admiración mutua y amistad entre el poeta y escritor indio Rabindranath Tagore y la escritora argentina Victoria Ocampo. El proyecto, además de constituir un valioso aporte humanista por su tratamiento del pensamiento de Tagore, es una clara evidencia de la búsqueda de ambas regiones por rescatar y representar los elementos que sus historias comparten. La amistad entre Tagore y Vijaya, como el poeta llamaba a la escritora, pues, es una de las instancias históricas de diálogo entre nuestras regiones que ha dejado importantes huellas en las culturas de ambas. La representación cinematográfica de esta historia, según creemos, llevada a cabo colaborativamente entre Argentina e India, no puede sino acercar las distancias que nuestro imaginario aún pone entre ambas regiones. ${ }^{26}$

En fin, si tenemos en cuenta la coyuntura global descrita que se presenta en la actualidad, podemos afirmar que los estudios de la India en Latinoamérica están atravesando una recontextualización respecto de sus antecedentes. Este nuevo contexto puede propiciar que ellos se desarrollen en un escenario más fértil y con finalidades de mayor alcance, y favorecer el estrechamiento y la multiplicación de lazos académicos. ${ }^{27}$ Esto implicaría el diseño de programas de intercambio de profesores y estudiantes, el establecimiento de proyectos investigativos de cooperación internacional, el ofrecimiento de cursos de grado internacionales, el dictado de programas de educación a distancia, etc. Asimismo, una característica relevante que este desarrollo manifiesta es la conciencia regional que los especialistas latinoamericanos en estudios de la India han comenzado a manifestar. Esto ha dado lugar al establecimiento de

24. Singh (2012: 69).

25. Leite (2012: 65).

26. Cfr. http://www.telam.com.ar/notas/201512/131244-cine-pablo-cesar-pensando-en-el-rabindranath-tagore-victoria-ocampo.html El cineasta desarrolla, asimismo, una intensa actividad de promoción de la cooperación cinematográfica Sur-Sur. En el último Festival Internacional de Cine de India, por ejemplo, organizado por la Federación de la Cámara de Comercio e Industria de India, presentó un Workshop sobre "Cine y Cooperación Sur-Sur”. Para un estudio pormenorizado de la influencia de la literatura india en la literatura y filosofía argentinas véanse Gasquet (2015), además de sus trabajos previos, y Cattedra (2015) entre otros de sus escritos.

27. No deben desestimarse, no obstante, las orientaciones políticas en auge en ambas regiones y la incidencia que estas pueden tener sobre las investigaciones producidas en las áreas de ciencias sociales y humanidades. Cfr. Solís (2001, pp. 891-900). 
una red, podríamos decir, de estudiosos latinoamericanos sobre India, lo que constituye una diferencia respecto de los trabajos que pioneros han tenido que desarrollar anteriormente percibido a nivel mundial como caracterizado por un gran nivel de aislamiento en la región. ${ }^{28}$ La Asociación de Estudios de Asia y África (Aladaa) ha tenido un enorme impacto en la formación de esta red acercando a especialistas que han integrado sus perspectivas en publicaciones y en trabajos colaborativos como el que ofrecemos. ${ }^{29} \mathrm{El}$ camino para el establecimiento de una escuela latinoamericana de estudios de la India es todavía incipiente pero parecería que las condiciones internas y la coyuntura global son propicias para transitarlo y para proporcionarle al área una mayor elasticidad en cuanto a su relativa autonomía académica.

\section{Unidades académicas en América Latina (parcial o totalmente) dedicadas a los estudios de la India ${ }^{30}$}

\section{Argentina}

- Área de Asia y África de la Facultad de Ciencias Sociales, Universidad Nacional de Lomas de Zamora.

- Cátedra de Estudios Indorientales, Universidad Abierta Interamericana.

- Cátedra de Historia Contemporánea de Asia y África, Universidad Nacional de Córdoba.

- Cátedra de Sánscrito, Facultad de Filosofía y Letras, Universidad de Buenos Aires.

- Cátedra India, Cámara Indo Argentina de Comercio - UCES, Argentina.

- Cátedra Libre de la India, Instituto de Relaciones Internacionales, Universidad Nacional de La Plata.

- Cátedra Mundo Actual Afroasiático, Facultad de Humanidades, Universidad Nacional de Comahue.

- Centro de Estudios de Asia y África, Facultad de Filosofía y Letras, Universidad de Buenos Aires.

- Centro de Estudios de Asia y África, Facultad de Filosofía y Letras, Universidad Nacional de Tucumán.
- Especialización en Economía y Negocios con Asia del Pacífico e India, Universidad de Tres de Febrero.

- Fundación Instituto de Estudios Budistas (FIEB), Argentina.

- Escuela de Estudios Orientales, Facultad de Filosofía, Letras y Estudios Orientales, Universidad del Salvador.

- Grupo de Estudios sobre India de Rosario - Programa PRECSUR, Universidad Nacional de Rosario.

- Grupo Darśana de Filosofía de la India y Filosofía Comparada, Instituto de Filosofía, Facultad de Filosofía y Letras, Universidad de Buenos Aires.

- Museo Nacional de Arte Oriental, Ministerio de Cultura.

Brasil

- Área de Língua e Literatura Sánscrita da Universidade de São Paulo.

- Centro de Estudos Afro-Asiáticos, Especialización: África; Asia; Relaciones de los países de la región con Brasil, Universidade Candido Mendes.

- Centro de Estudos Afro-Orientais, Especialización: Estudios afro-brasileños; lenguas y civilizaciones africanas y asiáticas, Universidade Federal Da Bahia.

- Centro de Estudos Asiáticos, Especialización: Asia y la relación de los países de la región con Brasil, Universidad de Brasilia.

- Departamento de Ciência da Religião da Universidade de Juiz de Fora.

- Fundação de BrIndARC - Brasil-Índia Associação de Redes de Conhecimento.

- Grupo de Trabalho Filosofia Oriental (AMPOF).

- Núcleo de Estudios Asiáticos, Centro de Estudios Avanzados Multidisciplinares, Universidad de Brasilia.

- Programa de Estudos Indianos. Universidade do Estado do Rio de Janeiro.

- Programa de Pos-graduação em Ciência da Religião, Universidade Federal de Juiz de Fora.

28. Véase nota 4 .

29. La Asociación cuenta con más de tres décadas de publicaciones relativas al área que contienen trabajos de especialistas latinoamericanos y del mundo. Véase también el reciente volumen de Rodríguez de la Vega, L. y Lavolpe, F. (2015). Estos autores cuentan, asimismo, con un antecedente colaborativo regional pero dedicado a una temática de más amplio espectro: Rodríguez de la Vega, L. y Lavolpe, F. (2014).

30. La presente lista es una etapa preliminar de una base de datos en preparación. Si desea aportar información acerca de otra unidad académica por favor comuníquese con Gabriel Martino por mail a la dirección gabriel.filosofia@hotmail.com 
- Programa de Pós-Graduação em Ciências das Religiōes da Universidade Federal da Paraíba

\section{Chile}

- Programa de Estudios Asiáticos, Especialización: Culturas asiáticas, Pontificia Universidad Católica de Chile.

\section{Colombia}

- Centro de Estudios sobre India Contemporánea, Universidad Externado de Colombia.

\section{México}

- Centro de Estudios Asiáticos, Especialización: todos los países asiáticos, énfasis en China, India, Japón y Corea, Universidad Autónoma de Nuevo León.

- Centro de Estudios de Asia y África (Colmex).

- Cátedras de Historia de la India, Literatura de la India y Filosofía de la India, Facultad de Filosofía y Letras, Universidad Autónoma de México.

\section{Venezuela}

- Centro de Estudios de África, Asia y Diásporas Latinoamericanas y Caribeñas "José Manuel Briceño Monzillo", Facultad de Humanidades y Educación, Universidad de Los Andes (ULA).

\section{Perú}

- Instituto de Estudios Clásicos Orientales y Occidentales, Especialización: Pensamiento, arte y letras de culturas orientales, Universdad Ricardo Palma, Lima.

- Centro de Estudios Orientales, Pontificia Universidad Católica del Perú.

\section{Referencias}

Beigel, F. (2010). La institucionalización de las ciencias sociales en América Latina: entre la autonomía y la dependencia académica. En F. Beigel (Ed.), Autonomía y dependencia académica. Universidad e investigación cientifica en un circuito periférico: Chile y Argentina (1950-1980). Buenos Aires: Biblos.

Beigel, F. (ed.) (2013). The Politics of Academic Autonomy in Latin America. Ashgate: Burlington.

Beigel, F., \& Salatino, M. (2015). Circuitos segmentados de consagración académica: las revistas de Ciencias So- ciales y Humanas en la Argentina. Información, Cultura y Sociedad, 32, 11-36.

Burges, S. (2012). Response to Questionnaire on India and Latin America. FPRC Journal, 3, 19-24.

Cattedra, O. (2015). La tradición india en el pensamiento de Francisco García Bazán. En B. Nantey \& L. Pinkler (Comp.), Gnosis y tradiciones sagradas. Buenos Aires: El hilo de Ariadna.

Facchini, G., Olarreaga, M., Silva, P., \& Willmann, G. (2010). Substitutability and Protectionism: Latin America's Trade Policy and Imports from China and India. The World Bank Economic Review, 24(3), 446-473.

Gasquet, A. (2015). El llamado de Oriente. Historia del orientalismo argentino (1900-1950). Buenos Aires: Eudeba.

Gaur. M., \& Gaur, I. eds. (2012). India and Latinoamerica, número especial del Foreign Policy Research Centre Journal, Vol. 3.

Heine, J. (2012). Response to Questionnaire on India and Latin America. FPRC Journal, 3, 5-11.

Heine, J., \& Viswanathan, R. (2011). The Other BRIC in Latin America: India. En Americas Quarterly: The New Brazil and the Changing Hemisphere, no. 5. no. 2 (Spring).

Lederman, D., Olarreaga, M., \& Perry, G. E. (2009). China’s and India's Challenge to Latin America. Opportunity or Threat? WA: The World Bank.

Leite, E. (2012). Brazil-India University Interchange. FPRC Journal, 3, 58-67.

Martino, G. (2015). Cuestiones en torno a la filosofía de la India. Tendencias académicas en las universidades de Argentina y dilemas (meta)filosóficos. Journal de Ciencias Sociales, 3(5), 1-22.

Mesquita-Moreira, M. (2012). Response to Questionnaire on India and Latin America. FPRC Journal, 3, 12-14.

Narayanan, R. (1983). Latin American Studies in India. Latin American Research Review, 18(3), 179-184.

Perry, G. (2006). Latin America and the Caribbeans Response to the Growth of China and India: Overview of Research Findings and Policy Implications. World Bank.

Rodriguez de la Vega, L., \& Lavolpe, F. (2014). Diversidades: Asia y África en perspectivas desde América del Sur. Buenos Aires: UNLZ.

Rodriguez de la Vega, L., \& Lavolpe, F. (2015). Perspectivas Sobre La India: Pasados y Presentes. Buenos Aires: UNLZ.

Rubiolo, M. F., \& Baroni, P. A. (2014). El rol de las economías emergentes en el siglo XXI: el caso de India en América Latina y su vinculación con Argentina. Temas y debates, 7(18), 101-121.

Shidore, S. (2013). New Frontiers in South-South Engagement. Relationship between India and Latin America of the 
Caribbean. New Delhi: Indian Council of World Affairs, Sapru House.

Singh, P. (2012). A New Era in India-Latin America Relations: Symptoms and Substance. FPRC Journal, 3, 68-75.

Solís, B. P. (2001). Las relaciones entre México y la India, 19952000. Foro Internacional, 41(4), 891-900.

Solís, B. P. (2008). Las relaciones entre México e India, 20002006. Foro Internacional, 48 (2), 487-493.
Tharoor, S. (2012). India-Latin America Relations: A Work in Progress. Georgetown Journal of International Affairs, 13(2), 69-74.

Tripathi, R. (2012). Sixty Years of Sanskrit Studies (1950-2010). New Delhi: Rashtriya Sanskrit Sansthan. 